\title{
BEHAVIOR OF GAS EMBOLI SUBJECTED TO PRESSURE VARIATION IN BIOLOGICAL SYSTEMS*
}

\author{
K. S. CHAN† and WEN-JEI YANG \\ The University of Michigan, Ann Arbor, Mich. 48104, U.S.A.
}

\begin{abstract}
Dynamic behavior of gas emboli in a quiescent liquid having the rheological properties of human or animal blood subject to pressure variations is studied. Consideration is given to step and sinusoidal changes in the system pressure. It is disclosed that in the study of dynamic behavior of gas emboli, a Newtonian model may be used to approximate the theological behavior of human or animal blood in which the emboli are situated. The instantaneous embolus size in blood under pulsating pressure change may be determined by the equilibrium condition of instantaneous static pressures.
\end{abstract}

\section{INTRODUCTION}

DURING open-heart surgery, microbubbles of air and oxygen in the extracorporeal circulation can enter the artificial circulation system and result in gas embolization which causes various degrees of harm to vital organs (Selman et al., 1967; Anderson et al., 1965; Spence et al., 1965; and Kaplan et al., 1962). The principal source of microbubbles is the blood channelled from the heart which is returned to an oxygenerator as an air-blood mixture. Venous air embolism is a recognized complication of various surgical (such as neurosurgical) diagnostic and therapeutic procedures. It may occur during anesthesia resulting from accidental injection or aspiration of large amounts of air into a vein (Durant et al., 1947; Michenfelder et al., 1966; Gottlieb et al., 1965; Munson et al., 1966; and Mayrhofer et al., 1966).

In the present work, the dynamic behavior of gas emboli situated in a quiescent liquid having the rheological properties of human or animal blood subject to pressure variations is studied. Experimentally measured shear stress-shear strain relationship of the blood of a dig, which is commonly supposed to have the properties of human blood, is used in the analysis. Consideration is given to sinusoidal and step changes in the system pressure. Results obtained from the study may cast light on the answer to two important questions: the possible growth or decay of an embolus during intracorporeal circulation of blood and the validity of analytical results using a Newtonian model for blood.

The study of bubble behavior subject to a step change and to sinusoidal changes of pressure has been reported by many investigators (Plesset, 1949; Forster et al., 1954; Plesset et al., 1954; Scriven, 1960; Borotnikova et al., 1964; and Solomon et al., 1967). However, in almost all those cases. the properties of the fluid, the magnitude of the pressure change and the frequency of the sinusoidal fluctuation are far different from those found in a biological system. In the present analysis, the shear stress-shear strain relationship of the fluid is as given by Bayliss (1960), the density and surface tension as given by Gottlieb et al. (1965), the frequency of the system pressure fluctuation is $72 \mathrm{c} / \mathrm{min}$, and the amplitude of fluctuation 0.05 . For the step change, the system pressure suddenly drops, at time equal to zero, from $1 \mathrm{~atm}$ to $\frac{1}{2} \mathrm{~atm}$. The initial embolus radius is $10^{-2} \mathrm{~cm}$

*First received 20 A ugust 1968; in revised form 16 October 1968.

†Fulbright-Hays visiting scholar from the University of Malaya.

$\ddagger$ Associate Professor of Mechanical Engineering. 
and the gas in the embolus undergoes an adiabatic process during the growth or collapse of the embolus. The effect of approximation with a Newtonian fluid or inviscid flujd on the result of analysis involving blood is also considered.

\section{ANALYSIS}

In spherical co-ordinates and on the assumption of a homogeneous incompressible liquid surrounding the embolus, the continuity and momentum equations can be written (Yang et al., 1966) as

$$
\frac{1}{r^{2}} \frac{\partial}{\partial r}\left(r^{2} u\right)=0
$$

and

$$
\rho_{l}\left(\frac{\partial u}{\partial t}+u \frac{\partial u}{\partial r}\right)=-\frac{\partial P}{\partial r}-|\nabla \cdot \bar{q}|_{r}
$$

respectively, where

$$
\left|\nabla . \overline{\tau_{1}}\right|_{r}=\frac{1}{r^{2}} \frac{\partial}{\partial r}\left(r^{2} \tau_{r r}\right)-\frac{\tau_{\theta \theta}+\tau_{\phi \phi}}{r} .
$$

If $R$ and $\dot{R}$ represent the instantaneous bubble radius and its first time derivative, integration of equation (1) from $r=R$ to $r=r$ yields:

$$
u=\frac{R^{2} \dot{R}}{r^{2}}
$$

When the last expression is substituted into equation (2) followed by integration between limits $r$ and $r_{0}$ it gives the following equation for the pressure distribution in the liquid.

$$
\begin{gathered}
\ddot{R} R^{2}\left(\frac{1}{r}-\frac{1}{r_{0}}\right)+\dot{R}^{2}\left(\frac{R^{4}}{2 r_{0}^{4}}-\frac{R^{4}}{2 r^{4}}+\frac{2 R}{r}-\frac{2 R}{r_{0}}\right) \\
=\frac{P_{l}(r)-P_{l}\left(r_{0}\right)}{\rho_{l}}-\frac{1}{\rho_{l}} \int_{r}^{r_{q}}|\nabla \cdot \overline{\bar{\tau}}|_{r} \mathrm{~d} r
\end{gathered}
$$

where $r_{0}$ is the reference radius from the embolus center in the liquid.

For the embolus surface, substitution of $R$ for $r$ and consideration for the equilibrium of forces on the bubble surface yields, as in Yang et al. (1966):

$$
\begin{gathered}
\rho_{l}\left[\ddot{R} R^{2}\left(\frac{1}{R}-\frac{1}{r_{0}}\right)+\dot{R}^{2}\left(\frac{R^{4}}{2 r_{0}^{4}}-\frac{2 R}{r_{0}}+\frac{3}{2}\right)\right] \\
=P_{g}(R)-P_{l}\left(r_{0}\right)-\frac{2 \sigma}{R}-4 \mu_{g} \frac{\dot{R}}{R}-\tau_{r r^{\prime} l}(R) \\
-\int_{R}^{r_{0}}|\nabla \cdot \overline{\bar{\tau}}|_{r} \mathrm{~d} r
\end{gathered}
$$

in non-dimensional form.

The shear stress $\tau_{\pi^{\prime} l}(R)$ and the integral in equation (5) for various fluids are given in Yang et al. (1966). Yang et al. (1965) presents an approximate method for the analysis of bubble dynamics in an incompressible, nonNewtonian liquid having an arbitrary stressstrain relationship. Briefly, if the shear stress-shear strain curve is approximated by $(n+1)$ linear combinations, $0,1,2, \ldots n$, and the slope of each is $\mu, \eta_{1}, \eta_{2}, \ldots \eta_{n}$, bubble equation (5) can be expressed as:

$$
\begin{aligned}
\rho_{l}\left(R \ddot{R}+\frac{3}{2} \dot{R}^{2}\right)= & P_{g}(R)-P_{l}(\infty)-\frac{2 \sigma}{R} \\
& -4\left(\eta_{n}+\mu_{0}\right) \frac{\dot{R}}{R} \mp 2 \sqrt{ }(3) \\
& \times\left(\frac{1}{3} \tau_{n}+\sum_{i=0}^{n-1} \tau_{i} \ln \frac{r_{i}}{r_{i}-1}\right)
\end{aligned}
$$

where the upper sign is for $\dot{R} \geqslant 0$, lower sign for $\dot{R}<0$

$r_{i}=\left(2 \sqrt{ }(3) R^{2}|\dot{R}| / C_{i}\right)^{1 / 2}$ for $i=0,1, \ldots$,

and

$$
n
$$

$$
C_{\mathrm{i}}=\frac{\mathrm{d} u}{\mathrm{~d} y} .
$$

The corresponding pressure distribution equation in the liquid at distance $r$ from the embolus center is: 


$$
\begin{aligned}
P_{l}(r) & =P_{l}(\infty)+\left[\frac{\ddot{R} R}{r}+R^{2}\left(\frac{2 \dot{R}}{r}-\frac{R^{4}}{2 r^{4}}\right)\right] \\
& \pm 2 V(3)\left(\tau_{n} \ln \frac{r_{n}}{r}+\sum_{i=0}^{n-1} \tau_{i} \ln \frac{r_{i}}{r_{i}+1}\right)
\end{aligned}
$$

The initial conditions are $R(0)=1 \cdot 0$, and $\dot{R}(0)=0$.

For the gas in the embolus undergoing a polytropic process, its pressure may be expressed as:

$$
P_{g}=P_{g}(0)\left[\frac{R(0)}{R(t)}\right]^{3 y}
$$

where $P_{g}$ is identically $P_{g}(R)$ in equation (6), $P_{g}(0)$ is the initial gas pressure and $\gamma$ is the polytropic constant. The two variations in the system pressure under consideration are: slowly applied to the embolus. Hence. the instantaneous mean bubble radius can be determined using the equation $R=2 \sigma / P_{y}$ $P(\infty)$ obtained by the equilibrium condition of static pressures, where $P(\infty)$ is the instantaneous blood pressure and $\sigma$ is the surface tension.

Numerical calculation was performed for the case in which the external pressure acting upon a human body undergoes a step change from one atmosphere to one half atmosphere. This corresponds to the case of $P(\infty)=P_{0}$ $(1+A \sin \Omega t+D)$ where $P_{0}=760 \mathrm{~mm} \mathrm{Hg}$. $D=-0.5, A=0.05$, and $\omega=72 \mathrm{c} / \mathrm{min}$. The shear stress-shear strain relationship for blood flowing in a rigid tube shown in Fig. 1 was used from which the parameters of a corresponding Bingham plastic was obtained. Results are illustrated in Figs. 2 and 3. The

$$
P_{l}(\infty)=\left\{\begin{array}{l}
P_{0}(1+A \sin \Omega t) \ldots \text { for a sinusoidal change } \\
P_{0}(1+A \sin \Omega t+D) \ldots \text { for a step change }
\end{array}\right.
$$

where $P_{0}$ is the atmospheric pressure, $P_{0} A$ is the amplitude of blood pressure pulse, $\Omega$ is the dimensionless frequency of blood pressure pulse and $P_{0} D$ is the amount of a step change in the external pressure.

\section{RESULTS AND DISCUSSION}

For a human body under normal atmospheric conditions, the blood pulse frequency is about $72 \mathrm{c} / \mathrm{min}$ or $\mathbf{0 . 0 0 0 7} \mathrm{rad}$. per unit of nondimensional time. The natural frequency of an embolus of radius $10^{-2} \mathrm{~cm}$ in human blood is about $19 \cdot 5 \mathrm{rad}$. per unit of non-dimensional time. Therefore, relative to the natural frequency of the embolus, the frequency of the blood pressure fluctuation is very slow. Under this condition, the embolus will oscillate at its natural frequency. This implies that as the blood pressure undergoes one cyclic change, the embolus oscillates as many times as its own natural frequency. Therefore, the effect of the blood pressure fluctuation on the embolus is like that of blood pressure sudden fall of the mean external pressure causes the sudden expansion of the gas in the embolus. The embolus then oscillates about its instantaneous mean radius corresponding to the instantaneous blood pressure. Its frequency is not the natural frequency at initial blood pressure which is 19.5 rad. per unit of nondimensional time but that corresponding to the subsequent instantaneous blood pressure.

Analogous to the previous case, it can be concluded that the effect of blood pressure pulsation on the dynamic behavior of the embolus is negligible. Through physical reasoning, one may find that the conclusion is also applicable to the case where the gas in the embolus is soluble in blood.

Figure 2 shows the time history of the embolus radius following a step change in the blood pressure at a large distance from the embolus. Because of the presence of gas in the embolus, the embolus size oscillates with time. Its amplitude decreases slightly but 


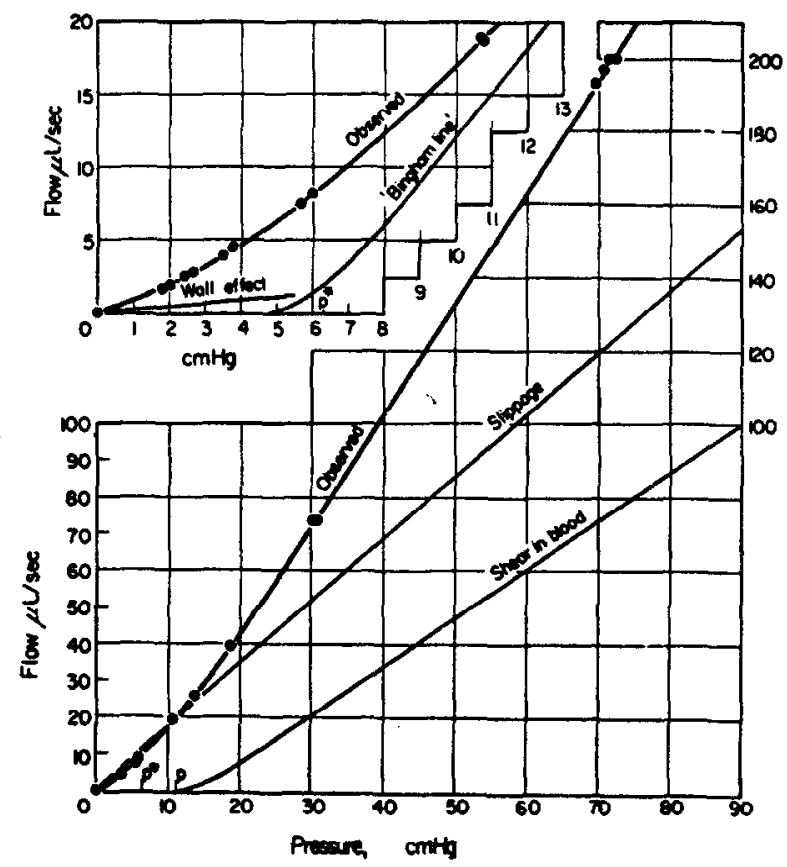

Fig. 1. The pressure-flow curve of dog's defibrinated blood, relative cell volume 0.49 , observed in a tube of radius $0.048 \mathrm{~cm}$ and length $155 \mathrm{~cm}$.

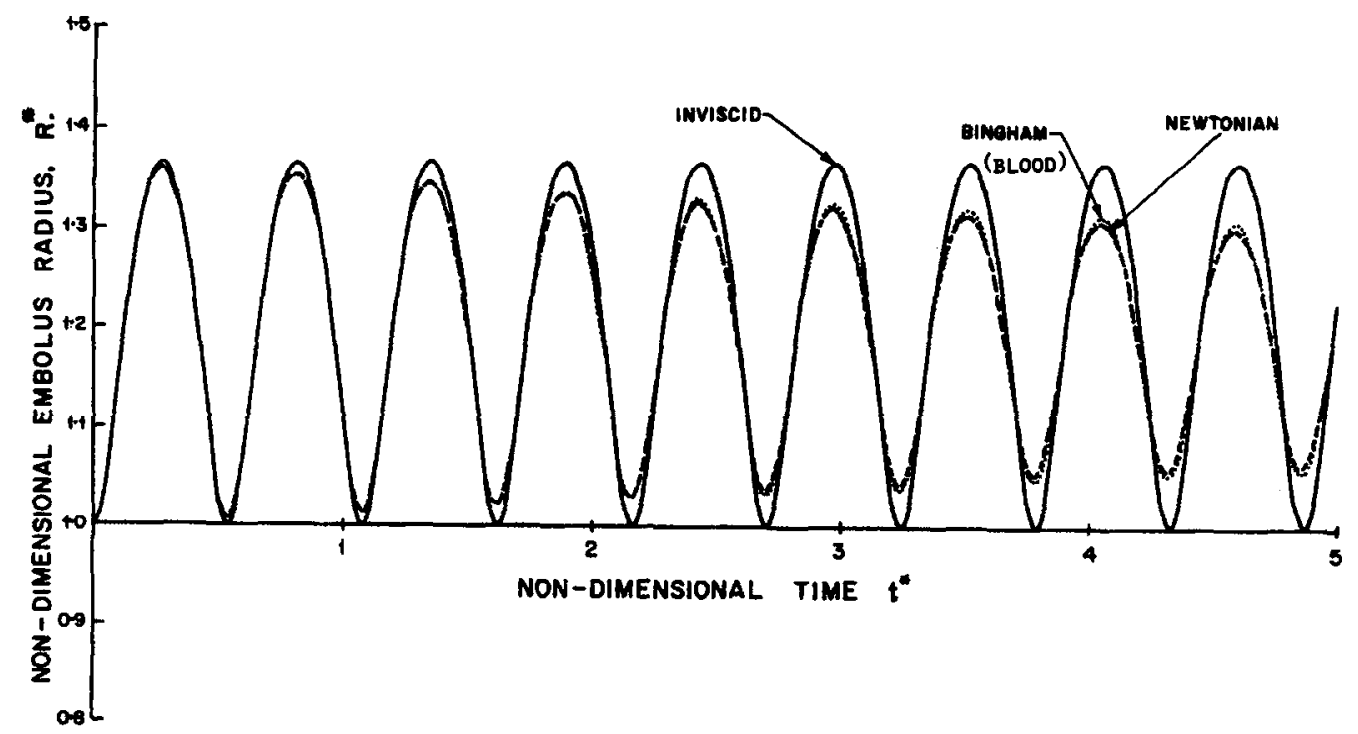

Fig. 2. Time history of embolus radius following a step change in system pressure from 1 atm to $0.5 \mathrm{~atm}$. 


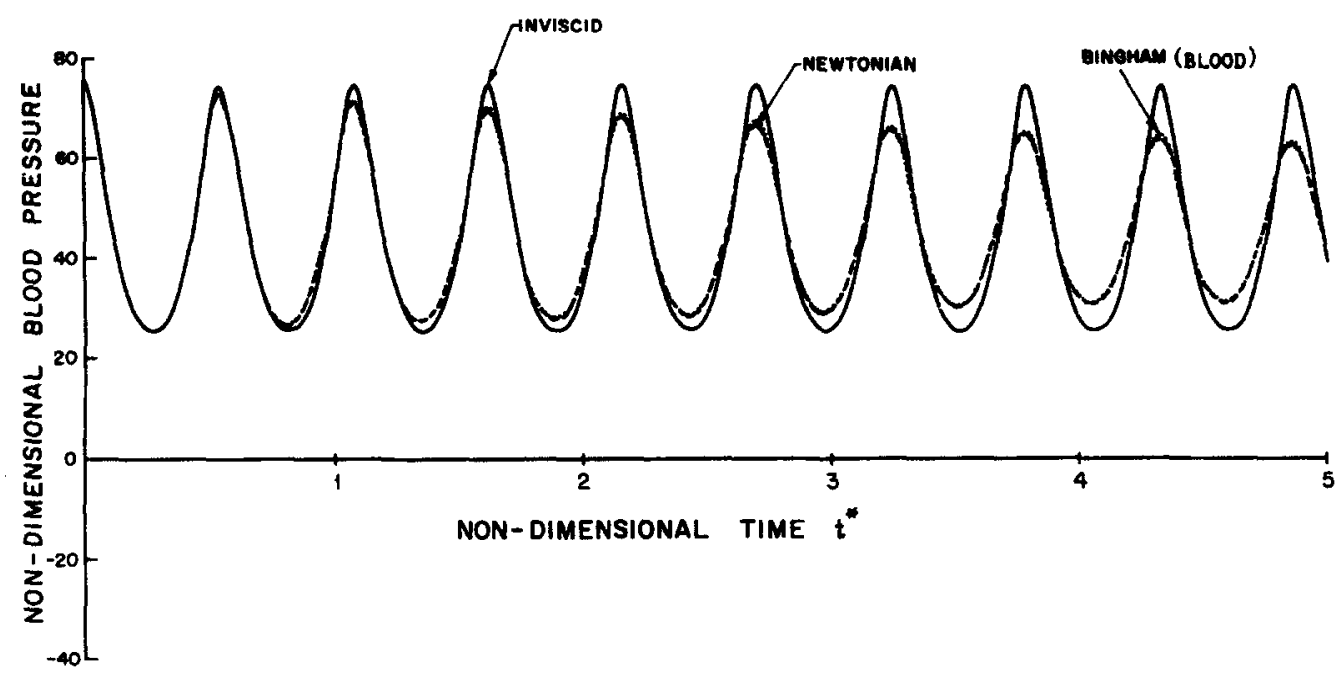

Fig. 3. Time history of blood pressure at $r=1.5 R_{0}$ following a step change in system pressure from 1 atm to $0.5 \mathrm{~atm}$.

continuously due to energy dissipation (irreversible conversion of mechanical energy into internal energy due to viscosity). Eventually, the oscillation will vanish leaving the embolus at a size consistent with the final system pressure.

In the absence of viscosity, blood will behave like an inviscid fluid. For such a case the oscillating amplitude remains constant as shown in Fig. 2. A significant difference in the dynamic behavior of the gas embolus resulting from viscous and non-viscous models for blood becomes obvious. Figure 2 also shows that the difference in results using a Newtonian Model or a Bingham Plastic Model to express the rheological behavior of blood is very small. This is because in the Bingham Model the yield stress $\tau_{0}$ is small, and so the shear stressshear strain curve can just as well be approximated by a Newtonian model. Hence, for practical purposes, a Newtonian fluid may be assumed.

Figure 3 gives the blood pressure fluctuation in the liquid at $r=1.5 R_{0}$ from the embolus center following a step change in the system pressure. The curves are similar, though of opposite sign, to those of radius oscillation shown in Fig. 2. Since the magnitude of local blood pressure falls rapidly away from the embolus center, the actual blood pressure in the immediate vicinity of the embolus can be expected to be much higher. In a manner analogous to the time history of the embolus radius, the amplitude of the blood pressure oscillation decreases with time for both the Bingham and Newtonian models. In the absence of viscosity, however, the oscillating amplitude will remain the same.

Acknowledgement-This investigation is supported by the Michigan Heart Association for which the authors are grateful.

\section{REFERENCES}

Alman. P. L. (1961) Biological handbooks: blood and other body fluids. Fedn. Am. Soc. exp. Biol. 12.

Anderson, R. M. et al. (1965) Pulmonary air Emboli during cardiac surgery. $J$. thorac. cardiovasc. Surg. 49, 440-449.

Bayliss, L. E. (1960) Flow Properties of Blood and Other Biological Systems. (Edited by A. L. Copley and G. Stainsky), p. 36. Pergamon Press, Oxford.

Borotnikova, M. I. and Soloukhin, R. 1. (1964) A calculation of the pulsations of gas bubbles in an incompressible liquid subject to a periodically varying pressure. Soviet Phys. Acoust, 10, 38-32.

Durant, T. M. Long, J. and Oppenheim, M. J. (1947). Pulmonary (venous) air embolism. Am. Heart J. 33, 269-281. 
Gottlieb. J. D., Ericcson, J. A. and Sweet, R. B. (1965) Venous air embolism, Anesth. \& Analg. 44, 773-779.

Kaplan, S., et al. (1962) Oxygen embolization during deep hypothermia. Archs. Surg. 84, 122-129.

Mayrhofer, O. and Steinbereithner. K. (1966) Significance of severe respiratory acidosis in pulmonary embolism during anesthesia. Anesth. \& Analg. 45, 564-568.

Michenfelder, J. D., et al. (1966) Air embolism during neurosurgery. Anesth. \& Analg. 45, 390-395.

Munson, E. S. and Merrick. H. G. (1966) effect of nitrous oxide on venous air embolism. Anesthesiology 27. 783-787.

Plesset, M. S. (1949) The dynamics of cavitation bubbles. J. appl. Mech. 16, 277-282.

Plesset, M. S. and Zwick, S. A. (1954) The growth of a vapor bubble in superheated liquids. J. appl. Phys. 25, 493-500.

Scriven, L. E. (1954) On the dynamics of phase growth. Chem. Engng Sci. 10, 1-13.

Selman, M. W., et al. (1967) The effectiveness of various heart-lung machines in the elimination of microbubbles from the circulation. J. thorac. cardiovasc. Surg. 53, 613-617.

Spencer, F. C., et al. (1965) The significance of air embolism during cardiopulmonary bypass. J. thorac. and cardiovasc. Surg. 49, 615-634.

Soloman, L. P. and Plesset, M. S. (1967) Nonlinear bubble oscillations. Calif. Inst. Tech. Rep. No. 85-38.

Yang. Wen-Jei and Yeh, Hsu-Chieh (1966) Theoretical study of bubble dynamics in purely viscous fluids. A.I.Ch.E.Jl12,927-931.

Yang, Wen-Jei and Yeh, Hsu-Chieh (1965) Approximate method for the determining of bubble dynamics in non-newtonian fluids. Physics Fluids 8, 758-759.

\section{NOMENCLATURE†}

$n, m$ parameters in power law model

$p$ pressure; $P_{o}(R)$, of gas at embolus surface; $\boldsymbol{P}_{00 .}$ of gas inside at zero time; $\boldsymbol{P}_{\mathrm{l}}(\boldsymbol{r})$, of blood, $P_{(}\left(r_{0}\right)$, of blood at a reference radius $r_{0} ; P_{10}(r)$, of blood at zero time; $\boldsymbol{P}_{(\infty)}$, of blood at infinity or system pressure.

pressure difference, $=P_{(\infty)}-P_{00}$

$$
\begin{aligned}
p^{*} \frac{p(r)-p_{00}}{\Delta p} ; p_{0}^{*}= & \frac{p_{0}(R)-p_{g 0}}{\Delta p} ; p_{l}^{*}= \\
& \frac{p(r)-p_{g o}}{\Delta p} ; p_{i 0}^{*}=\frac{p_{i 0}(r)-p_{g o}}{\Delta p}
\end{aligned}
$$

$R$ embolus radius

$\boldsymbol{R}_{\mathbf{0}}$ initial embolus radius

$R \quad \mathrm{~d} R / \mathrm{d} t$

$R \quad \mathrm{~d}^{2} R / \mathrm{d} t^{2}$

$R^{*} \quad R / R_{0}$

$\dot{R}^{*} R(\rho / \Delta p)^{1 / 2}$

$\ddot{R}^{*} \quad R R_{0} \rho_{l} / \Delta p$

$r$ distance from the center of spherical embolus

$r_{0}$ reference radius

$t$ time

$t^{*}\left(t / R_{0}\right)\left(\Delta p / \rho_{l}\right)^{1 / 2}$

$u$ radial velocity of blood flow at $r$.

\section{Greek letters}

$\eta$ parameter of Bingham model

$\mu$ viscosity; $\mu_{l}$, of liquid; $\mu_{g}$, of gas

$\mu^{*} \quad\left(\mu / R_{0}\right)(1 / \rho, \Delta p)^{1 / 2}$

$\rho$ density; $\rho_{l}$, of blood; $\rho_{g}$ of gas

$\sigma$ surface tension

$\sigma^{*} \sigma /\left(R_{0} \Delta p\right)$

$\tau$ normal stress; $\tau_{r r}, \tau_{\theta \theta}$ and $\tau_{\phi \phi}$, in the direction of $r, \theta$ and $\phi$, respectively

$\tau_{0}$ yield stress of Bingham model

$|\nabla . \nexists|_{r}$ viscous force per unit volume in radial direction

$\omega$ frequency of blood pressure pulsation

$\omega_{n}$ natural frequency of embolus radius oscillation in blood

$\Omega \quad \omega R_{0}\left(\Delta p / \rho_{l}\right)^{-1 / 2}$

$\Omega_{n} \quad \omega_{n} R_{0}\left(\Delta p / \rho_{l}\right)^{-1 / 2}$

\section{Superscripts}

* dimensionless physical quantity

- first derivative with respect time

.. second derivative with respect time.

\section{Subscripts}

$g$ gas

$l$ blood

0 at zero time, except $r_{0}$ and $\tau_{0}$.

†Unit in GGS system. 